Association for Information Systems AIS Electronic Library (AISeL)

Wirtschaftsinformatik Proceedings 2003

Wirtschaftsinformatik

September 2003

\title{
Konstruktion und Anwendung fachkonzeptioneller Referenzmodelle im Data Warehousing
}

Jörg Becker

Westfälische Wilhelms-Universität Münster, becker@wi.uni-muenster.de

Ralf Knackstedt

WestfälischeWilhelms-Universität Münster, israkn@wi.uni-muenster.de

Follow this and additional works at: http://aisel.aisnet.org/wi2003

\section{Recommended Citation}

Becker, Jörg and Knackstedt, Ralf, "Konstruktion und Anwendung fachkonzeptioneller Referenzmodelle im Data Warehousing" (2003). Wirtschaftsinformatik Proceedings 2003. 74.

http://aisel.aisnet.org/wi2003/74

This material is brought to you by the Wirtschaftsinformatik at AIS Electronic Library (AISeL). It has been accepted for inclusion in Wirtschaftsinformatik Proceedings 2003 by an authorized administrator of AIS Electronic Library (AISeL). For more information, please contact elibrary@aisnet.org. 
In: Uhr, Wolfgang, Esswein, Werner \& Schoop, Eric (Hg.) 2003. Wirtschaftsinformatik 2003: Medien - Märkte - Mobilität, 2 Bde. Heidelberg: Physica-Verlag

ISBN: 3-7908-0111-9 (Band 1)

ISBN: 3-7908-0116-X (Band 2)

(C) Physica-Verlag Heidelberg 2003 


\title{
Konstruktion und Anwendung fachkonzeptioneller Referenzmodelle im Data Warehousing
}

\author{
Jörg Becker, Ralf Knackstedt \\ Westfälische Wilhelms-Universität Münster
}

\begin{abstract}
Zusammenfassung: Fachkonzeptionelle Referenzmodelle liefern Ausgangslösungen für die Erstellung projektspezifischer Fachkonzepte und können damit einen Beitrag zur Erhöhung der Effizienz und Effektivität eines Data-WarehouseProjektes leisten (Abschnitt 1). Innerhalb des Data Warehousing sind bisher wichtige, die Formulierung von Modellvarianten unterstützende Konstruktionskonzepte für Referenzmodelle weitgehend unberücksichtigt geblieben (Abschnitt 2). Konfigurationsmechanismen ermöglichen die Ableitung von Referenzmodellvarianten in Abhängigkeit von Parametern, die den Anwendungskontext des Referenzmodells beschreiben. Weil konfigurierbare Referenzmodelle dadurch besonders passgenaue Ausgangslösungen bereitstellen können, schlägt der Beitrag die Erweiterung fachkonzeptioneller Data-Warehouse-Modellierungstechniken um Konfigurationsregeln vor und ordnet die Anwendung der Spracherweiterungen in ein umfassendes Phasenmodell der konfigurativen Referenzmodellierung ein (Abschnitt 3). Die Sicht des Referenzmodellanwenders wird bei der Einordnung der Referenzmodellkonfiguration in den Kontext von Data-Warehouse-Entwicklungsprojekte eingenommen (Abschnitt 4). Mit einem Fazit endet der Beitrag (Abschnitt 5).
\end{abstract}

Schlüsselworte: Data Warehousing, Fachkonzeption, Referenzmodellierung, Konfiguration, Modellvariantenmanagement

\section{Nutzenpotenziale fachkonzeptioneller Referenzmodelle}

Informationsmodelle, die als Ausgangslösungen zur Entwicklung projektspezifischer Modelle Verwendung finden, werden als Referenz-Informationsmodelle (im Folgenden kurz: Referenzmodelle) bezeichnet [Broc02, S. 42-45]. Mit der Topologie von Informationsmodellen [Schü98, S. 63-73] haben Referenzmodelle gemeinsam, dass sie sich unterschiedlichen Entwicklungsphasen der Informationssystemgestaltung, z. B. in der Aufteilung Fachkonzept, DV-Konzept, Implementierung [Sche98, S. 38ff.], zuordnen lassen. Aufgrund der Vielzahl von Ansätzen, welche die Transformation fachkonzeptioneller Data-Warehouse-Modelle in DV- 
konzeptionelle Schemata und Implementierungen behandeln [Hahn02; Herd01; Blas00; Holt99], wird im Folgenden der Schwerpunkt auf die Bereitstellung von Ausgangslösungen für die Fachkonzeption gelegt.

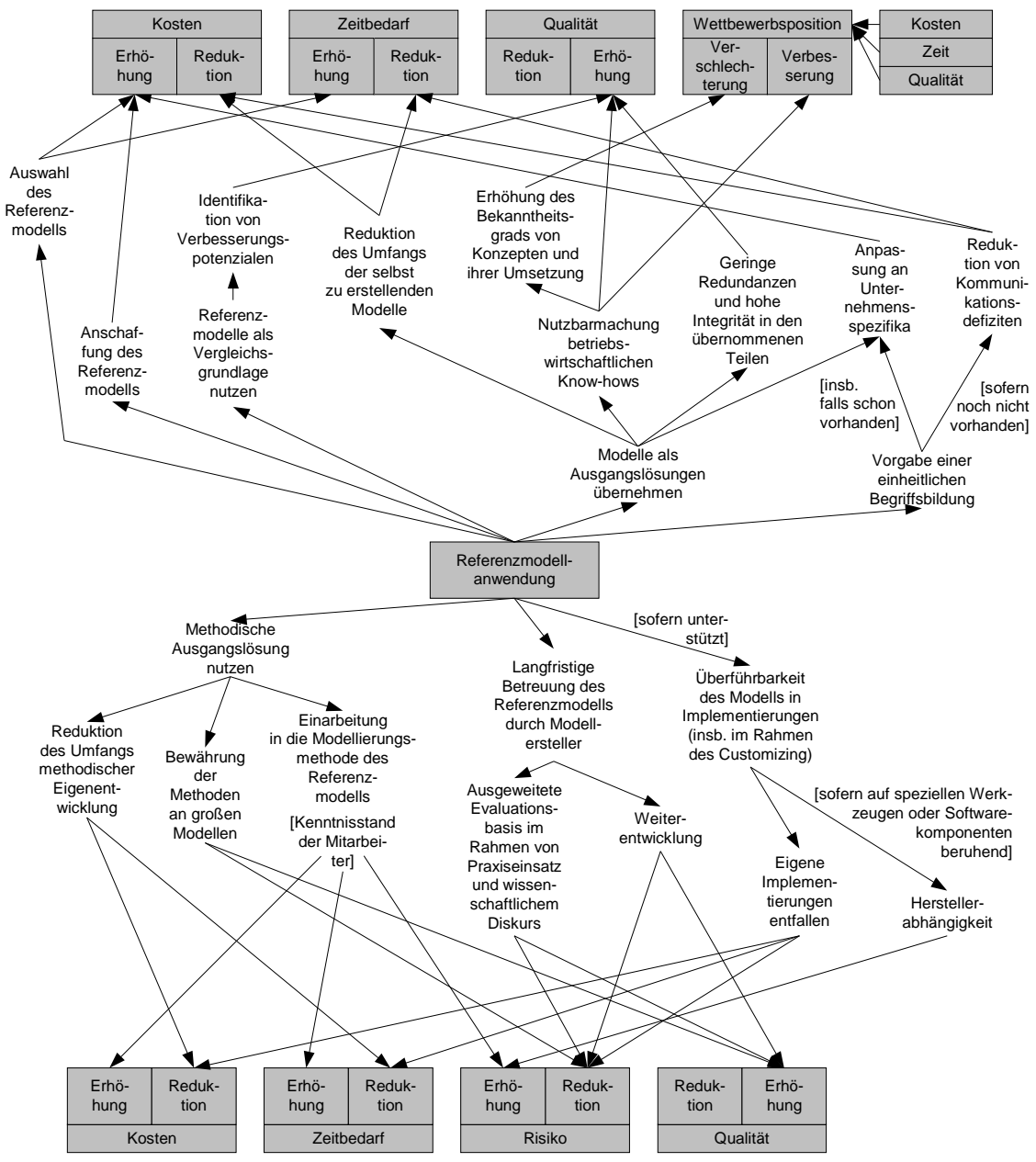

Abbildung 1: Wirkungen der Anwendung fachkonzeptioneller Referenzmodelle

Referenzmodelle schaffen für Anwender und Ersteller Nutzenpotenziale [BADK02]. Auf der Anwenderseite sollen sie die Wirtschaftlichkeit der Informationsmodellierung erhöhen (vgl. ausführlich Abbildung 1), ${ }^{1}$ indem sie Vergleichsgrundlagen zur Beurteilung der eigenen Lösungen schaffen und übernehmbare

1 Die Nutzenpotenziale der Referenzmodellierung werden in der Regel argumentativ hergeleitet [Simo98, S. 100ff.]. Empirische Untersuchungen liegen kaum vor bzw. fuBen auf unzureichenden Fallzahlen [Schü98, S. 74-80; Maie96]. 
Modellteile und Begriffssysteme bereitstellen. Darüber hinaus bieten sie Orientierung bei der methodischen Gestaltung der eigenen Modellsysteme. Den Vorteilen steht der Aufwand der Auswahl und Anschaffung der verwendeten Ausgangsmodelle sowie der Anpassung des Modells an projektspezifische Besonderheiten gegenüber.

Aus der Sicht der Referenzmodellersteller können Referenzmodellen die folgenden Rollen zukommen:

- Referenzmodelle können als Instrumente des Wissensmanagements interpretiert werden, da sie eine Möglichkeit zur Explikation von Gestaltungsempfehlungen darstellen. Besonders für Institutionen, die wiederholt ähnliche Projekte durchführen bzw. begleiten, kann die Entwicklung und interne Nutzung von Referenzmodellen lohnend sein.

- Das Referenzmodell kann darüber hinaus als selbstständiger Umsatzträger fungieren.

- Steht das Referenzmodell als Input für ein Modellierungstool bereit, kann es als gewichtiges Kaufargument für diese Werkzeug dienen.

- Referenzmodelle können zudem als Akquiseinstrumente für Beratungsaufträge eingesetzt werden, wovon neben Forschungsinstitutionen vor allem Beratungshäuser Gebrauch machen.

- Das Referenzmodell kann als Basis für die Anpassung von Softwaresystemen an den betrieblichen Anwendungskontext genutzt werden. Referenzmodelle dieser Art werden auch als Software-Referenzmodelle bezeichnet. Hersteller großer Enterprise Resource Planing (ERP)-Systeme haben dieses modellbasierte Customizing sehr erfolgreich umgesetzt.

Die vielfältigen Nutzenpotenziale für Modellanwender und -ersteller motivieren ihren Einsatz auch in der Domäne der Fachkonzeption von Data-WarehouseSystemen [GaG197, S. 37]. Deshalb wird im Folgenden der State-of-the-Art des Referenzmodelleinsatzes im Data Warehousing betrachtet und auf der Basis erkannter Defizite werden Entwicklungsvorschläge unterbreitet.

\section{State-of-the-Art fachkonzeptioneller Referenzmodellierung}

Eine im Rahmen von Data Warehousing weit verbreitete Form von Referenzmodellen stellen verschiedene Architekturmodelle von Data-Warehouse-Systemen dar [BaGü01, S. 31-36], die dessen Softwarekomponenten unterscheiden und diese in der Regel in verschiedene Schichten (z. B. Quellen, Import, Datenhaltung, Präsentation \& Analyse) gruppieren und zueinander in Beziehung setzen. Die Ori- 
entierung an diesen DV-konzeptionellen Modellen ist weitaus ausgeprägter als die Diskussion um die Wiederverwendung fachkonzeptioneller Modelle. Vorgehensmodellen zur Entwicklung von Data-Warehouse-Systemen [StWi02; Matt96; Poe96], wie sie aktuell vielfältig vorgeschlagen werden, fehlen auffällig häufig Aufgabentypen, die der Auswahl, der Anschaffung und der Anpassung von Referenzmodellen gewidmet sind [FeLo02, S. 9-10]. Wesentlich vorangetrieben wurde die fachkonzeptionelle Referenzmodellierung dagegen vor allem durch Arbeiten, die der Geschäftsprozessmodellierung zugerechnet werden können. Die dort entwickelten Ansätze lassen sich durch die - auch kombiniert einsetzbaren - Mechanismen, mittels derer der Referenzmodellanwender bei der Anpassung des Referenzmodells an projektspezifische Anforderungen unterstützt wird, unterscheiden [Broc02, S. 319ff.]. Die Mechanismen planen und begrenzen die Modellanpassungen des Anwenders in unterschiedlichem Ausmaße (vgl. Abbildung 2).

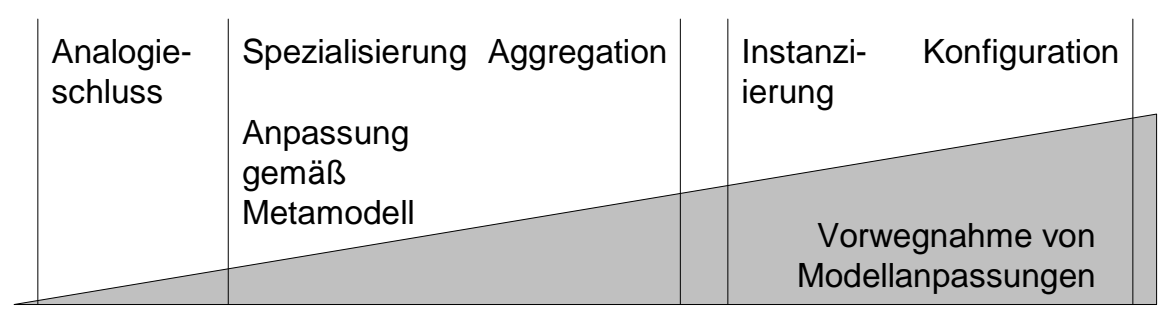

Abbildung 2: Unterstützung der Referenzmodellanwendung

Ist das Referenzmodell Gegenstand von Analogieschlüssen, bestehen keine Beschränkungen und Vorgaben, welche Teile des Modells in welcher Form übernommen werden. Zusammenstellungen von Entwurfsmustern verfolgen diese Strategie [Risi00; FeYu00]. Eine Beschränkung des Freiheitsgrades der Modellanpassung erhält man, wenn Modellmodifikationen wie die Löschung, Umbenennung oder Ergänzung von Modellteilen den Definitionen des Metamodells des Referenzmodells gerecht werden müssen [Hars94]. Dies gilt auch für Referenzmodelle, die mittels Spezialisierung angepasst werden sollen. Sie unterscheiden sich von den projektspezifischen Modellen insbesondere durch einen höheren Abstraktionsgrad, der sich häufig auch durch eine geringere Anzahl von Modellelementen ausdrückt [Roh195; BeSc96]. Die Aggregation erfordert, dass das Referenzmodell nicht als monolithischer Block vorliegt, sondern in Bausteine gegliedert ist, die von dem Referenzmodellersteller zu neuen Lösungen zusammengesetzt werden können. Die Kombinierbarkeit der Bausteine wird durch die Schnittstellendefinitionen der Bausteine determiniert [Lang97]. Eine noch stärkere Lenkung der Anpassungsmaßnahmen kann durch Platzhalter vorgenommen werden, die der Referenzmodellanwender durch zulässige Ausprägungen des Wertebereichs der Platzhalter zu belegen hat [Remm97, S. 112ff.; Schw99]. Die Wertebereiche können sich dabei sowohl auf numerische oder alphanumerische Instanzen als auch auf ganze Teilmodelle beziehen. Die Konfiguration sieht dagegen vor, das Referenzmodell mit expliziten Anpassungspunkten zu versehen, die vorschreiben, wie das 
Referenzmodell in Abhängigkeit gewählter Konfigurationsparameterausprägungen anzupassen ist [BDKK02]. Die durch Automatisierbarkeit geprägte Konfiguration kann als Pol eines Kontinuums von Ansätzen zur Referenzmodellanpassung angesehen werden; ihr steht auf der anderen Seite die durch weitgehende kreative Freiheit charakterisierte Analogiekonstruktion gegenüber.

Der State-of-the-Art des Einsatzes fachkonzeptioneller Referenzmodelle im Data Warehousing ist - sofern eine Modellwiederverwendung überhaupt vorgenommen wird - schwerpunktmäßig durch die nicht vorgeplante modellierungsmethodenkonforme Modifikation von Ausgangsmodellen geprägt. Dies äußert darin, dass in den zahlreichen vorgeschlagenen Modellierungsmethoden zur fachkonzeptionellen multidimensionalen Modellierung keine Konstrukte zur Formulierung von Platzhaltern oder Konfigurationsansatzpunkten vorgesehen sind. Die von Inmon publizierten Referenzmodelle sind dem Ansatz der Spezialisierung zuzuordnen (vgl. www.billinmon.com). Parallelen zum Ansatz der Aggregation finden sich in der Bereitstellung von Bibliotheken wiederverwendbarer Elemente von fachkonzeptionellen Data-Warehouse-Referenzmodellen, auf die beim Aufbau neuer Modelle zurückgegriffen werden können. Die Ansätze sind zumeist auf spezielle Modellelementtypen spezialisiert [Spit97]. Auf eine lange betriebswirtschaftliche Tradition können dabei Sammlungen von Definitionen von Kennzahlen und Kennzahlensystemen verweisen [Reic97]. Allerdings vernachlässigen sie mit den zu untersuchenden Bezugsobjekten und die zu unterstützenden Managementaufgaben wichtige Aspekte der fachkonzeptionellen Spezifikation [BeHo98, S. 483]. Eine automatisierte Anpassung des Referenzmodells an Konfigurationsparametern ist vor allem im Rahmen von ERP-Systemen realisiert. Die als Konfigurationsparameter berücksichtigen Aspekte des Anwendungskontextes sind dabei zumeist auf die Auswahl vordefinierter Berichte oder die Selektion zu unterstützender Rollen von Berichtsempfänger beschränkt. Die Dokumentation der Konfigurationsregeln in fachkonzeptionellen Modellen ist zudem unzureichend unterstützt, da die Konfiguration durch Anpassung DV-konzeptioneller Strukturen erfolgt und den Fachanwendern lediglich die Auswirkungen in Form eliminierter Berichte bzw. Berichtsteile präsentiert werden.

Die Rezeption und Reflektion konfigurativer Referenzmodellierungsansätze in der Domäne des Data Warehousing wird vor dem Hintergrund des State-of-the-Art als defizitär empfunden. Im Folgenden werden methodische Erweiterungen vorgeschlagen, welche konfigurierbare Referenzmodelle für das Data Warehousing nutzbar machen sollen. 


\section{Konstruktion konfigurierbarer Referenzmodelle}

Die Erstellung konfigurierbarer Referenzmodelle lässt sich in fünf Phasen gliedern, die logisch aufeinander aufbauen (vgl. Abbildung 3) [BDKK02, S. 36]. Im Rahmen der praktischen Durchführung sind Rücksprünge in vorangegangene Phasen jederzeit erlaubt.

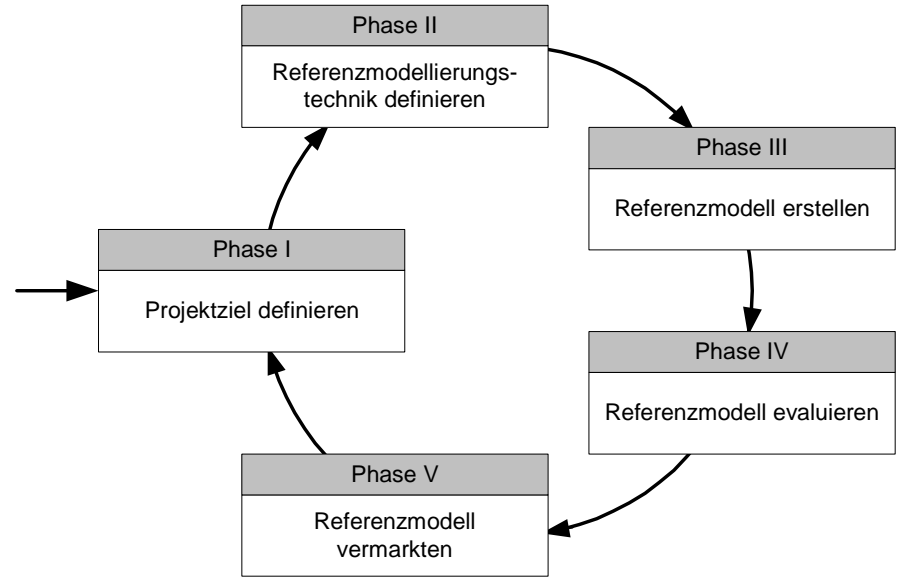

Abbildung 3: Referenzmodellkonstruktion

\section{Projektziel definieren}

Konfigurierbare Referenzmodelle zeichnen sich durch die Bereitstellung von Referenzmodellvarianten aus, die explizit beschriebenen Anwendungskontexten zugeordnet sind. Die Auswahl der zu unterstützenden Anwendungskontexte stellt einen wesentlichen Beitrag zur Einschränkung des Konstruktionsauftrages dar. Ansätze zur konfigurativen Referenzmodellierung unterscheiden sich insbesondere in der Art der Kontextspezifikation [FeLo02, S. 6-9]. Verbreitet ist der Rückgriff auf Unternehmensmerkmale, Perspektiven und Funktionsbereiche:

- Anhand von Unternehmensmerkmalsausprägungen werden Unternehmensklassen beschrieben. Entsprechende Topologien können verwendet werden, um den Zusammenhang zwischen Unternehmenseigenschaften und funktionalinhaltlichen Anforderungen an Informationssysteme zu beschreiben [MeLo00; MLEM99].

- Projektspezifische Besonderheiten, die sich aus beteiligten Benutzergruppen und verfolgten Zwecken der Referenzmodellanwendung ergeben, werden über Perspektiven berücksichtigt. Auffassungsunterschieden unterschiedlicher Modellnutzer (Perspektivendifferenzen [Woll86, S. 13]) sind auch Gegenstand der multiperspektiven Modellierung [Fran94; Rose98] und dem Domain-specific Modeling [NSKL98]. 
- Dependent zu der Abgrenzung von Unternehmensklassen und Perspektiven ist die Auswahl zu unterstützender Funktionsbereiche. Die Definition von Funktionsbereichen berücksichtigt, dass Referenzmodelle nicht zwangsläufig Ausgangslösungen für vollständige Unternehmensmodelle zur Verfügung stellen, sondern sich zum Beispiel auf Produktion oder Marketing spezialisieren [FeLo02, S. 18].

Bei der Festlegung des Projektziels sind als Restriktionen das vorhandene bzw. beschaffbare Know-how auf dem abgegrenzten Gebiet sowie das Marktpotenzial des zu schaffenden Referenzmodells zu berücksichtigen.

\section{Referenzmodellierungstechnik definieren}

Aus der Projektzieldefinition sind die Anforderungen an die Referenzmodellierungstechnik abzuleiten. Für die multidimensionale Modellierung von DataWarehouse-Fachkonzepten werden eine Vielzahl unterschiedlicher Modellierungstechniken vorgeschlagen [z. B. Bulo96; HeHa99; SBHD99; Sche98; Toto00]. Sie lassen sich zum Beispiel danach unterscheiden, ob sie sich eng an Entity-Relationship-Modelle anlehnen bzw. diese erweitern, aus dem Bereich der Scientific and Statistical Data Bases stammen, sich an objektorientierte bzw. erweitert objektorientierte Modellierungsansätze anlehnen oder unter Verzicht auf eine Anlehnung an bestehende Modellierungsansätze einen eigenen multidimensionalen Ansatz entwickeln [Böhn01, S. 156-160]. Neben terminologischen Differenzen und unterschiedliche Stärken und Schwächen in der detaillierten Definition einzelner fachkonzeptioneller Aspekte (z. B. Beschreibung rechentechnischer Zusammenhänge zwischen Kennzahlen, Festlegung alternativer Bezugsobjektstrukturen (Heterachien, Dimensionsausschnitte etc.), Formulierung von Historisierungsanforderungen) zeigen die Ansätze grundlegende Gemeinsamkeiten. Die Fachkonzeption zielt auf die Definition von aufgaben- und adressatengerechten Navigationsräumen durch Fakten (auch Multidimensionale Datenstrukturen genannt), wobei Fakten Kombinationen von Kennzahlen (auch Maßzahlen genannt) und Bezugsobjekten (auch Dimensionselemente genannt) darstellen. Die Navigation durch die Fakten wird durch drei Beziehungsarten beeinflusst [HoDS02, S. 423ff.]:

- Dimensionen ordnen Bezugsobjekte hierarchisch und geben damit Aggregations- bzw. Disaggregationspfade vor. Dimensionen, die auf der untersten Ebene die gleichen Bezugsobjekte aufweisen, können zu Dimensionsgruppen zusammengefasst werden.

- Kennzahlensysteme ordnen die zu betrachtenden qualitativen Aspekte der Bezugsobjekte rechentechnisch oder sachlogisch. Auch sie geben damit Analysepfade vor.

- Da bestimmte Berechnungsvorschriften (z. B. Abweichung des Ist-Umsatzes vom Vorjahr) sowohl Kennzahlen als auch Bezugsobjekte einbeziehen, sollten 
darüber hinaus auch auf Faktenebene Navigationspfade definiert werden können.

Für die konfigurative Referenzmodellierung bedarf es einer Erweiterung des sprachbasierten Metamodells [Stra96, S. 24] bestehender fachkonzeptioneller Data-Warehouse-Modellierungsmethoden. Die für die Konfiguration vorzusehenden Modellelementtypen der Modellierungsmethode müssen über Regeln mit Konfigurationsparametern in Beziehung gesetzt werden. Als Konfigurationsparameter dienen Unternehmensmerkmalsausprägungen und Perspektiven, die bereits im Rahmen der Projektzieldefinition identifiziert wurden oder aber im Rahmen der Modellerstellung erweitert bzw. verfeinert werden (vgl. Abbildung 4).

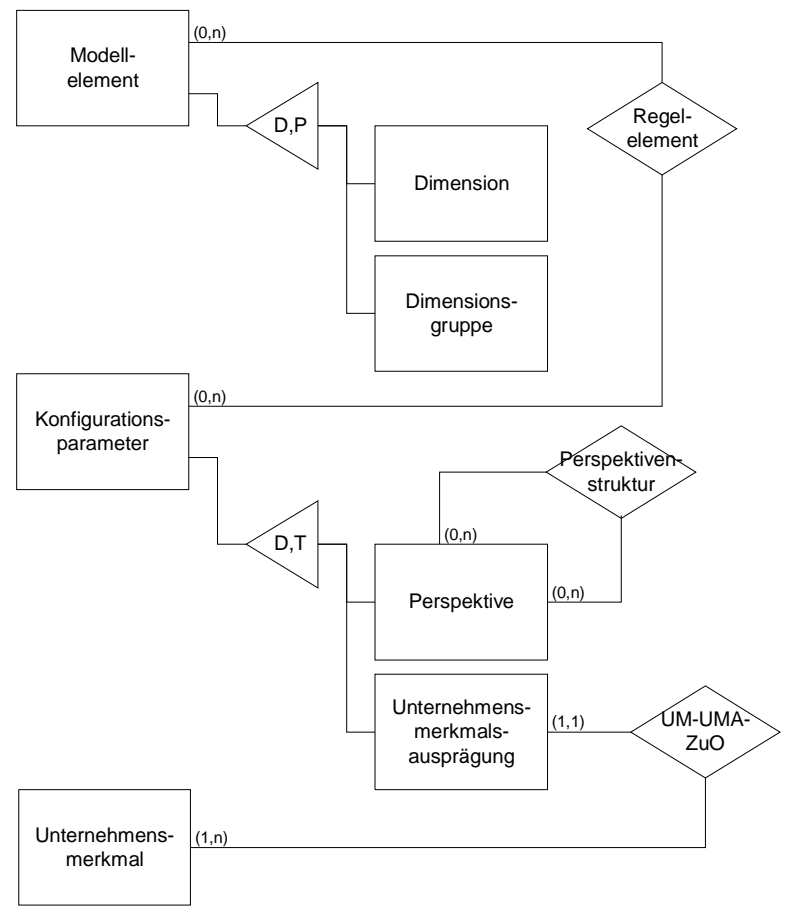

Abbildung 4: Metamodellerweiterung für die Konfiguration

Für die Regeln lassen sich alternative Repräsentationsformen unterscheiden (vgl. im Vorgriff auch Abbildung 7). Sogenannte Buildtime-Operatoren zeichnen sich dadurch aus, dass ihre Regelmenge mehrere einzelne Modellelemente betreffen können. Ihre Notation sollte daher für solche Modellelemente vorgesehen werden, die einzelne Modellelemente miteinander verknüpfen (z. B. Relationshiptypen in Entity-Relationship-Modellen [Schü98, S. 268]) bzw. einschließen (z. B. öffnende und schließende Konnektoren in ereignisgesteuerten Prozessketten [Schü98, S. 246]). Im Fall multidimensionaler Modelle sind die den definierten Navigationsraum bezeichnenden Modellelemente für Buildtime-Operatoren geeignet. Die dem 
Buildtime-Operator zugeordnete Regelbasis kann z. B. als Entscheidungstabelle dargestellt werden. Im alternativen Fall werden einzelne Modellelemente um Parametrisierungen ergänzt, die festlegen, bei Gültigkeit welcher Konfigurationsparameterausprägungen das jeweilige Modellelement Teil des abgeleiteten projektspezifischen Modells ist [Schw99, S. 143-148]. Die Syntax der Parametrisierungen kann in Form einer kontextfreien Grammatik festgelegt werden (vgl. Abbildung 5). Als Sprache für die Definition der kontextfreien Grammatik selbst wird (auf Meta-Metaebene) die erweiterte Backus-Naur-Form (EBNF) [Loud94, S. 83102] verwendet.

\begin{tabular}{|c|c|}
\hline $\begin{array}{l}<\text { Term> } \\
<\text { Ausdruck> } \\
\text { <Ausdruck> } \\
\text { <Perspektiven-Ausprägungsliste> } \\
\text { <Perspektiven-Ausprägungsliste> } \\
<\text { Unternehmensmerkmals-Ausprägungsliste> } \\
\text { <Unternehmensmerkmals-Ausprägungsliste> } \\
<\text { Operator } \\
<\text { Präfix> }\end{array}$ & 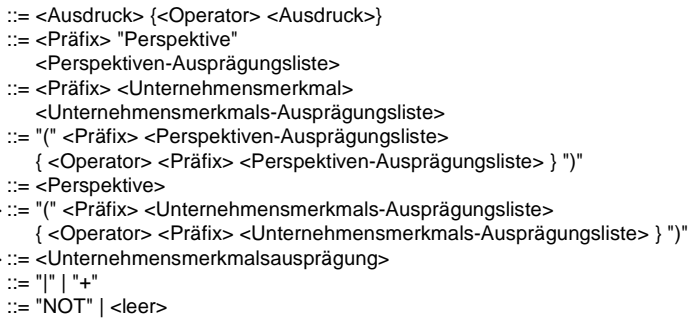 \\
\hline
\end{tabular}

Abbildung 5: Kontextfreie Grammatik in EBNF zur Formulierung von Parametrisierungen

Neben der Konfiguration einzelner Modellelemente sind darüber hinaus Konfigurationen größerer Granularität zu erwägen, die auch Anpassungen auf Metamodellebene vornehmen. Insbesondere für die Unterstützung perspektivenspezifischer Referenzmodellvarianten eignet sich die Definition von Modelltypen. Die Modelltypen (auch „,building blocks“ genannt) werden über Metamodelle definiert (vgl. Abbildung 6). In ihrer Gesamtheit beschreiben sie die methodische Informationssystemarchitektur [Espr89; Fran99; Zach87] des Referenzmodells. Die Berücksichtigung weiterer Modelltypen erlaubt es, den Adressatenkreis des DataWarehouse-Referenzmodells erheblich auszuweiten und kann damit die Effektivität seiner Nutzung steigern.

Für den multidimensionale Navigationsräume spezifizierenden Modelltyp bieten sich folgende Modelltypen als Ergänzung an:

- Die Navigationsräume können an Managementfunktionen in erweiterten Ereignisgesteuerten Prozessketten (EPK) [KeNS92] annotiert werden, um auszudrücken, im Rahmen welcher Entscheidungsfindungen die Navigationsräume analysiert werden [BeKn02, S. 136-145]. Im Rahmen der Organisationsgestaltung lässt sich so ein besserer Überblick über den Grad der Unterstützung der Geschäftsprozesse durch Informationssysteme gewinnen, und der Manager kann den Zweck des Data-Warehouse-Modells passender einordnen.

- In EPKs sind in der Regel Outputdaten von operativen Funktionen verzeichnet. In speziellen Modelltypen sollte die Zuordnung dieser operativen Daten zu den Navigationsräumen hergestellt werden. Dieser Modelltyp ist für den Gestalter 
des ETL-Prozesses des Data-Warehouse-Systems von herausragendem Interesse.

- Da die Navigationsräume auch mittels webbasiertem OLAP [Kurz99, S. 377ff.] zur Verfügung gestellt werden können, bietet sich eine Verknüpfung mit Referenzmodellen von Internetauftritten (site) an [Scha97]. Der Navigationsraum kann dabei der Spezifikation einer einzelnen Internetseite (page) zugeordnet werden, die in die Verknüpfungsstruktur der Referenz-Site eingeordnet ist. Auf diesem Wege lässt sich auch das Web-Applikationsdesign als Perspektive des Referenzmodells einbinden.

Darüber hinaus empfiehlt es sich zu berücksichtigen, dass Bezugsobjekte und Kennzahlen, die im Rahmen der Definition der Data-Warehouse-Navigationsräume spezifiziert werden, eine Untermenge der Begriffe darstellen, die der Content-Manager zur inhaltlichen Klassifikation seiner verschiedenen Objekte (Texte, Bilder, Videos, URLs etc.) verwendet [BeKS02]. Die Navigationsraummodelle lassen sich entsprechend um Modellelemente erweitern, die auch Untersuchungsobjekte und Aspekte dieser Untersuchungsobjekte beschreiben, die vom DataWarehouse-System nicht als Bezugsobjekte bzw. Kennzahlen verwaltet werden. Die erweiterten Modelltypvarianten (vgl. nochmals Abbildung 6) würden ContentManagern zugeordnet werden, während Data-Warehouse-Administratoren weiterhin die auf Bezugsobjekte und Kennzahlen beschränkte Modelltypvariante zur Verfügung gestellt bekämen.

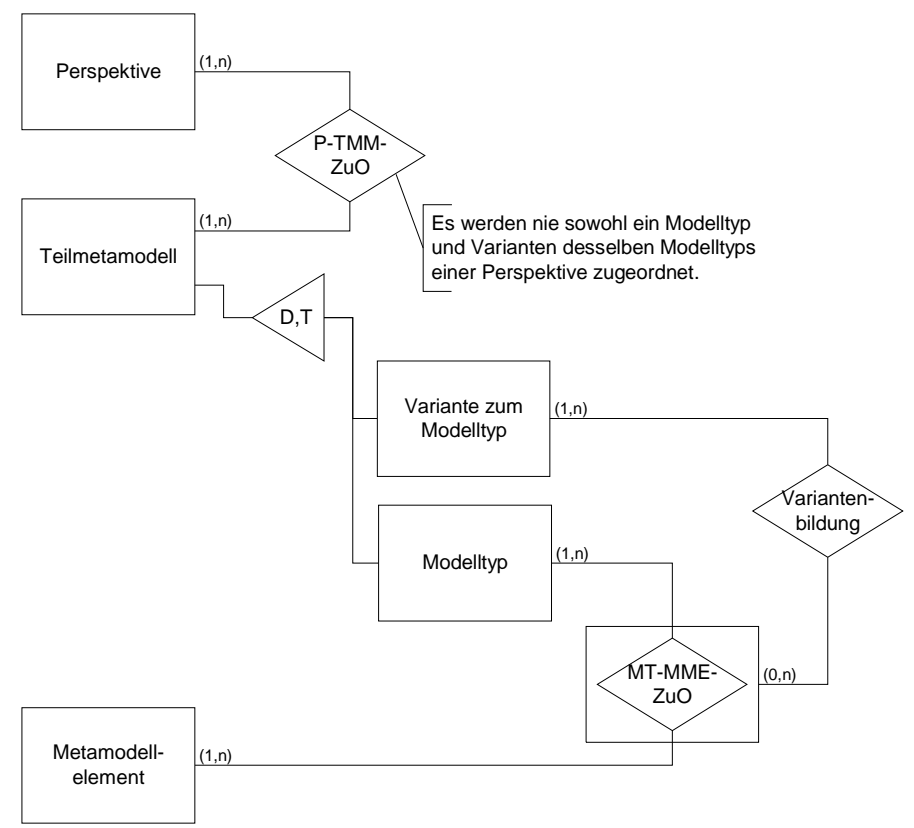

Abbildung 6: Modelltypen und Modelltypvarianten 
Weitere Konfigurationsmechanismen, die bei der Erweiterung der Modellierungstechnik von besonderem Interesse sind, sind die Bezeichnungsvariation und die Darstellungsvariation [BDKK02]. Die Bezeichnungsvariation ermöglicht einen von den Konfigurationsparametern abhängigen Austausch von Begriffen (z. B. die Ersetzung des Begriffs „Rechnung“ durch den Begriff „Faktura“). Die Darstellungsvariation ermöglicht die perspektivenspezifische Anpassung der Modelldarstellung, z. B. den Austausch von formalen Symbolen im Modell des Administrators gegen besonders intuitiv verständliche Piktogramme im Modell des Fachanwenders oder die Unterstützung spezifischer Modelltopologien (z. B. Spaltendarstellung von EPKs) zur Unterstützung spezieller Analysen.

\section{Referenzmodell erstellen}

Unter Verwendung der individuellen Referenzmodellierungstechnik ist das eigentliche Referenzmodell schrittweise zu konstruieren. Im Rahmen der Erstellung des Referenzmodells selbst muss eine Zuordnung der Ausprägungen der Konfigurationsmechanismen zu den Komponenten des Referenzmodells vorgenommen werden. Die Modellerstellung wird im Folgenden anhand eines Navigationsraums für die Artikel(gruppen)analyse exemplifiziert (vgl. Abbildung 7). Der Navigationsraum wird angegeben, indem $\mathrm{zu}$ analysierende Bezugsobjektdimensionen verschiedener Dimensionsgruppen und passende Kennzahlensysteme ausgewählt werden. ${ }^{2}$ Als Konfigurationsparameter werden die Unternehmensmerkmale Geschäftsart, Bezugsraum und Berichtszyklus betrachtet. Es ist davon auszugehen, dass eine Analyse der Artikelumsätze nach Aktionsarten nur sinnvoll ist, wenn auch der Geschäftprozess „Aktionsgeschäft“ betrieben wird und nicht z. B. eine Dauerniedrigpreis-Strategie verfolgt wird. Die Betrachtung der Artikel nach Herkunftsländern wird nur als sinnvoll angesehen, falls der Bezugsraum die Ausprägung ,international“ aufweist. Die Periodizität der Berichterstattung wirkt sich in der zu wählenden Auswertungshierarchie über Tage aus. Die dem BuildtimeOperator in Abbildung 7 zugehörige Entscheidungstabelle ordnet den genannten Bedingungen in Form von Kombinationen von Unternehmensmerkmalsausprägungen Aktionen zu. Die Aktionen bestehen im Entfernen bzw. Hinzufügen von Modellelementen. Die verwendeten Kreuze symbolisieren, dass das jeweilige Modellelement Bestandteil des abgeleiteten projektspezifischen Modells sein soll. Die alternative Verwendung von Parametrisierungen zeigt die Abbildung 7 anhand der an die Dimension „Artikelgruppe Herkunftsland“ notierte Regel „Bezugsraum (international)“, die festlegt, dass die Dimension bei ausschließlich nationaler Ausrichtung des Unternehmens entfällt.

2 Die Notation für Dimensionen und Kennzahlensystemen in Abbildung 7 basiert auf dem MetaMIS-Ansatz (vgl. z. B. [HoDS02] und den Beitrag von Holten, Bergerfurth und Becker in diesem Band). Eine detaillierte Modellierung von Navigationsräumen wird z. B. über die Definition von Dimensionsausschnitten und Ausdrücken für Berechnungen auf Faktenebene ermöglicht. 


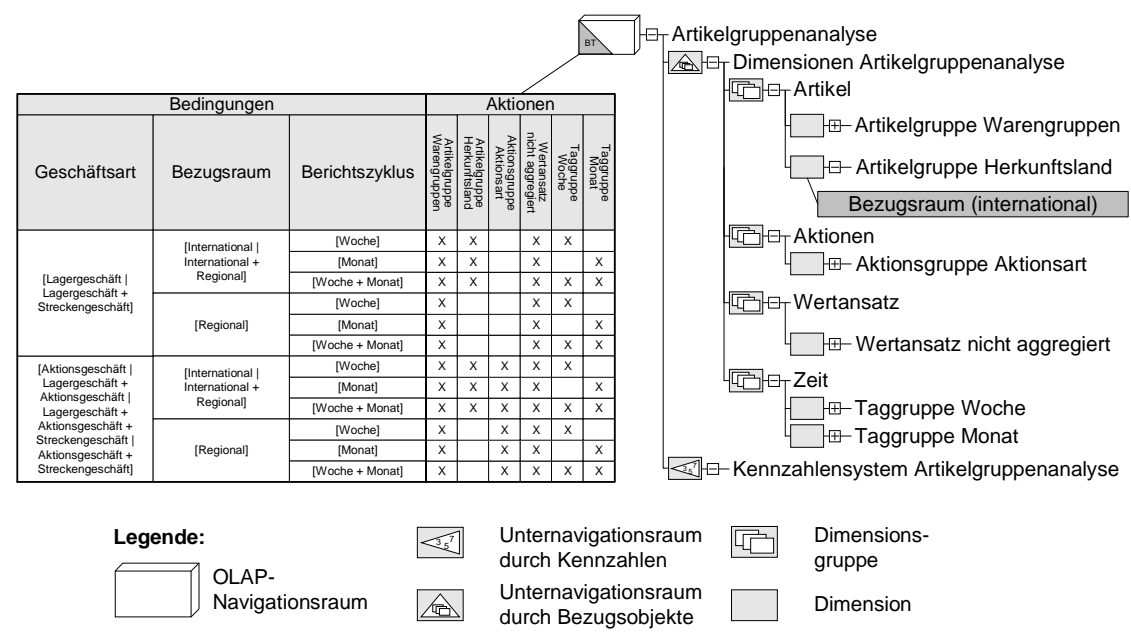

Abbildung 7: Konfigurierbares multidimensionales Modell

\section{Referenzmodell evaluieren}

Vor seiner Publikation ist das Referenzmodell als Ganzes abschließenden Tests zu unterziehen. Die Testfälle, die der Evaluation des Referenzmodells zugrunde liegen, stellen geeignet auszuwählende Kombinationen der Konfigurationsparameter (Konfigurationsvektoren) dar.

Im Rahmen der Evaluation ist insbesondere sicherzustellen, dass Interdependenzen zwischen einzelnen Konfigurationen korrekt berücksichtigt werden. Z. B. muss sich der Wegfall von datenerhebenden Funktionen im Prozessmodell auch in der Modifikation von Navigationsräumen widerspiegeln.

Referenzmodell vermarkten

Abschließend sind die Bedingungen (Distributionspolitik, Produktpolitik, Kommunikationspolitik, Kontrahierungspolitik [Meff91]) festzulegen, zu denen das Referenzmodell zugänglich gemacht wird. Die Bildung marktorientierter Modellvarianten zur Unterstützung einer differenzierten Produktpolitik kann sich ebenfalls ganz wesentlich auf die Auswahl von Perspektiven und Unternehmensmerkmalen stützen. 


\section{Anwendung konfigurierbarer Referenzmodelle}

Die Anwendung konfigurierbarer Referenzmodelle im Rahmen der Gestaltung von Data-Warehouse-Systemen erweitert das Aufgabenspektrum entsprechender Vorgehensmodelle (vgl. Abbildung 8). Die Problemdefinition des DataWarehouse-Projektes sollte bereits unter Rückgriff auf Unternehmensmerkmale, Perspektiven und Funktionsbereiche erfolgen, um in der nachfolgenden Phase die Suche und Selektion eines passenden konfigurierbaren Referenzmodells zu erleichtern.

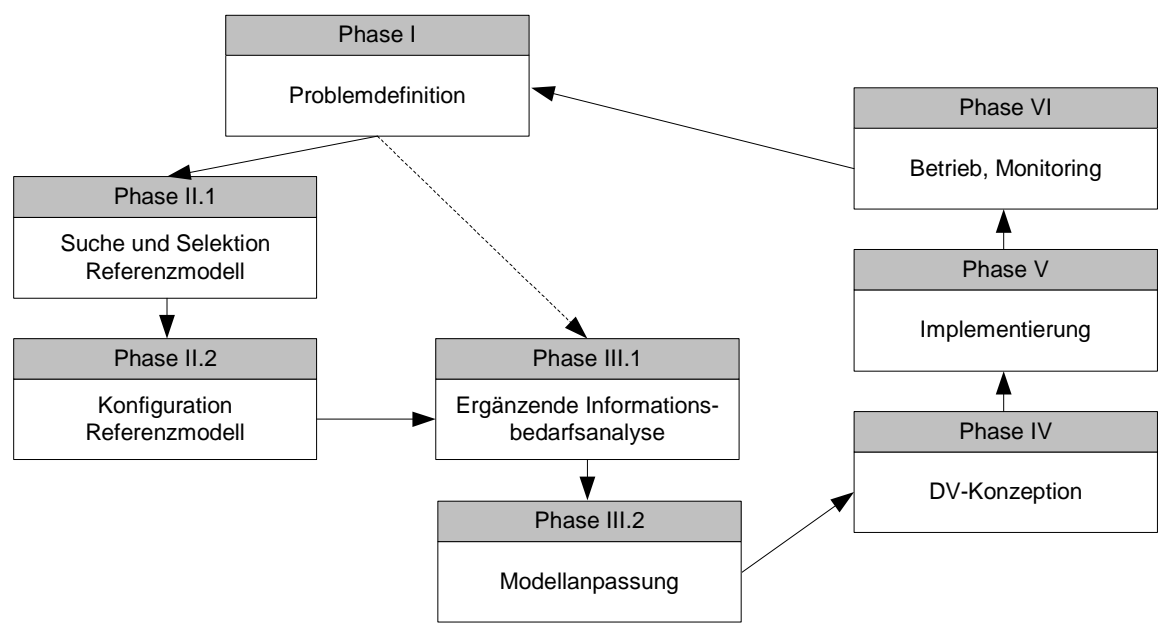

Abbildung 8: Referenzmodellanwendung

Zur Unterstützung der Suche und Selektion von Referenzmodellen wird der Aufbau und die Verwendung von Referenzmodellkatalogen vorgeschlagen [FeLo02]. Stehen entsprechende Kataloge zur Verfügung, kann die Identifikation geeigneter Referenzmodelle wesentlich erleichtert werden, indem die Anforderungen der Problemdefinition mit den zu den Modellen verwalteten Merkmalen verglichen werden. Zurzeit ist weder der Bestand an fachkonzeptionellen Referenzmodellen für das Data Warehousing befriedigend noch ist eine angemessene Transparenz über vorhandene Ausgangslösungen erzielt.

Die Konfiguration der Modelle selbst kann weitgehend automatisiert erfolgen. Hierfür eignet sich als eine mögliche Implementierungsalternative (neben z. B. rein datenbankgestützten Lösungen) die Formulierung der konfigurierbaren, fachkonzeptionellen Data-Warehouse-Modelle in Prolog [Knac01]. Zu Beginn der Konfiguration sind die Konfigurationsparameter zu erheben. In Abbildung 9 wird ein entsprechender Dialog für die Unternehmensmerkmale Geschäftsart, Bezugsraum und Berichtszyklus gezeigt (vgl. für die folgenden Beispiele auch nochmals Abbildung 7). Hier wird davon ausgegangen, dass der Dialog in einer prozedura- 
len Programmiersprache realisiert ist. Abbildung 9 (4) skizziert, wie eine Verbindung zwischen dem Dialogmodul und einer Inferenzmaschine (Prologserver) hergestellt werden kann. Nach Erhebung der Konfigurationsparameter wird die Inferenzmaschine über das Prädikat „liefereModellelemente“ aufgerufen, wobei die im vorgeschalteten Dialog ermittelten Parameter übergeben werden. Das Prädikat erstellt eine Liste aller Modellelemente (Variable X), die unter den übergebenen Konfigurationsparametern in das konfigurierte Modell zu übernehmen sind. Dafür werden in die Regelbasis zunächst zusätzliche Prädikate eingefügt (,asserta“), die die Gültigkeit der jeweiligen Parameter formulieren (vgl. Abbildung 9 (3)). Daraufhin wird das Prädikat „erstelleModellelementliste“ ausgewertet, das der eigentlichen Identifizierung der gültigen Modellvariante dient. Es verwendet das Metaprädikat „findall““, das nacheinander für Modellelemente prüft, ob sie in dem projektspezifischen Modell enthalten sind.

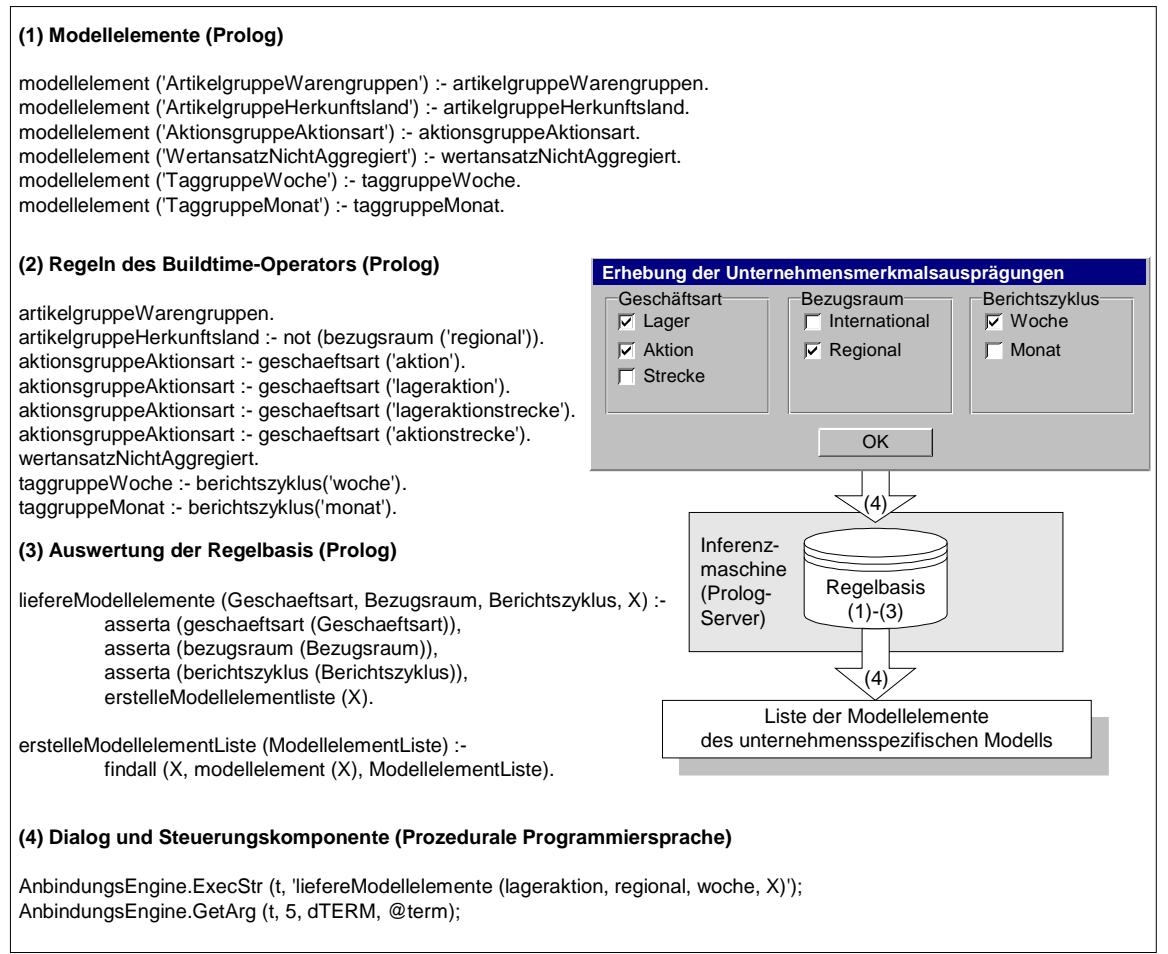

Abbildung 9: Konfigurationswerkzeug

Für die Prüfung der einzelnen Modellelemente auf Enthaltensein im konfigurierten Modell wird das Prädikat „,modellelement“" verwendet (vgl. Abbildung 9 (1)). Ist eine Klausel dieses Prädikats wahr, wird der Name des Modellelements an die Modellelementliste angehängt, die an die Variable X gebunden wird. Die Klauseln des Prädikats „modellelement“ sorgen insbesondere dafür, dass ein Modellele- 
mentname maximal einmal in die Liste aufgenommen wird. Die Entscheidung, ob ein Modellelement der Liste angefügt wird, erfolgt über die Auswertung der Prädikate der Konfigurationsregeln (vgl. Abbildung 9 (2)).

Nach Auswertung der Regelbasis steht die an die Variable X gebundene Modellelementliste in Form einer Zeichenkette zur Verfügung und kann ausgewertet werden (vgl. nochmals Abbildung 9 (4)). Mit der Auflistung der relevanten Modellelemente ist die im spezifischen Anwendungsfall zu verwendende Referenzmodellvariante identifiziert und kann zur grafischen Darstellung an einen geeigneten Editor übergeben werden.

Konfigurierbare Referenzmodelle können nicht sämtliche Projektspezifika berücksichtigen, was sich darin äußert, dass die Konfigurationsparameter aus Gründen der Komplexitätsbeherrschung und -reduktion einzelne Projektunterschiede clustern und damit von weiteren Besonderheiten abstrahieren. Daher muss das konfigurierte Data-Warehouse-Referenzmodell daraufhin überprüft werden, ob es den projektspezifischen Informationsbedarf korrekt wiedergibt. Bei dieser Prüfung können die bekannten Informationsbedarfsanalysemethoden zum Einsatz kommen [Stru97, S. 38-47]. Mit der Vorbereitung dieser Informationsbedarfsanalysen kann ggf. bereits parallel zur Referenzmodellanwendung begonnen werden (vgl. den gestrichelten Pfeil in Abbildung 8). Der Vorteil des Einsatzes des konfigurierten Referenzmodells liegt darin, dass bereits zu Beginn von Interviews oder Dokumentanalysen Ausgangslösungen vorliegen, die eine kritische Hinterfragung und Verbesserungsideen anregen. Häufig ist die Bereitstellung erster Lösungen Voraussetzung für eine effektive Befragung von Führungskräften.

Die Ergebnisse der Prüfung des konfigurierten Referenzmodells schlagen sich in Modellanpassungen nieder. Hierbei können die übrigen Konstruktionsmechanismen von Referenzmodellen (vgl. nochmals Abbildung 2) zum Einsatz kommen. Die Einhaltung methodischer Konventionen sollte durch Modellierungswerkzeuge unterstützt werden (Anpassung gemäß Metamodell). Um die Sicherstellung der Konsistenz zwischen Modellteilen zu unterstützen, sollten Modellelemente wie Dimensions- und Kennzahlendefinitionen in Bibliotheken verwaltet werden [HoKn99, S. 59-61; BHKU01, S. 90-91]. Bei der Erweiterung des Ausgangsmodells sollten die Modellelemente diesen Bibliotheken entnommen werden (Aggregation). Die Bibliotheken können dabei zweistufig gestaltet sein: Die erste Stufe beinhaltet initial alle Modellelemente des konfigurierten Referenzmodells, während die zweite Stufe auch diejenigen Modellelemente zur Verfügung stellt, die im Rahmen der Konfiguration eliminiert wurden. Insbesondere hinsichtlich detaillierter Vorgaben für Dimensionen empfiehlt es sich, den Konfigurationsansatz dahingehend zu erweitern, dass ausgewiesen wird, welche Bezugsobjekthierarchien lediglich exemplarischen Charakter haben und im Rahmen von Analogieschlüssen an Unternehmensspezifika anzupassen sind und welche Hierarchien wie z. B. die CCG-Warengruppenhierarchie [CCG97] tatsächlichen Referenzcharakter besitzen. 
Das angepasste fachkonzeptionelle Modell ist anschließend mittels mehrerer Modelltransformationen zu implementieren und bei Bedarf evolutionär an neue Anforderungen anzupassen.

\section{$5 \quad$ Fazit}

Die konfigurative Referenzmodellierung weist einige Besonderheiten auf, die es erstrebenswert erscheinen lassen, sie auch für das Data Warehousing zu erschließen: ${ }^{3}$

- Aufgrund der Explikation der Konfigurationsregeln ist die konfigurative Referenzmodellierung in besonderem Maße geeignet, vorhandenes Gestaltungswissen detailliert zu dokumentieren.

- Durch die Verwaltung unterschiedlicher Modellvarianten lässt sich die Passgenauigkeit der als Ausgangslösungen dienenden Modelle erhöhen, so dass der Anpassungsbedarf für den Nutzer reduziert und die Wirtschaftlichkeit des Referenzmodelleinsatzes erhöht werden kann (vgl. nochmals Abbildung 1).

- Zudem stellen die den Modellvarianten zugeordneten Konfigurationsparameterausprägungen ein Instrument zur Explikation des Modellkontextes dar, was den Auswahlprozess erleichtert [FeLo02] und zur Sicherstellung der kommunikativen Funktion des Modells beiträgt [Wolf01, S. 136ff.].

Referenzmodellierungsansätze zielen darauf ab, Gestaltungswissen in geschlossener, konsolidierter Form zu dokumentieren. Organisational-Memory-Systeme dienen dagegen der Dokumentation von Ergebnissen einzelner Projekte [HaTB02]. Mit ihnen lassen sich sowohl Projekterfolge als auch -mißerfolge beschreiben. Durch Abgleich mit aktuellen Anforderungen sollen Organisational-MemorySysteme zurückliegende Projekte hoher Verwandtschaft ermitteln. Entsprechende Ansätze stellen eine geeignete Ergänzung des hier verfolgten Ansatzes dar, weil die Projektdokumentationen zur Akquise des zu modellierenden Wissens eingesetzt werden können. Solange allerdings Fälle nicht in ausreichender Anzahl zur Verfügung stehen, erscheint eine induktive und deduktive Herleitung von Referenzmodellen über die Analyse einzelner erfolgreicher Data-WarehouseImplementierungen und die Heranziehung von Empfehlungen der betriebswirtschaftlichen Literatur [BeSc96, S. 49ff.] besonders Erfolg versprechend.

3 Eine empirische Prüfung dieser Wirkungshypothesen im Rahmen weiterführender Forschungen erscheint wünschenswert, setzt aber zunächst die Erarbeitung der Methodik und ihrer Werkzeugunterstützung voraus. 


\section{Literatur}

[BADK02] Becker, J.; Algermissen, L.; Delfmann, P.; Knackstedt, R.: Referenzmodellierung. WISU, 31 (2002) 11, S. 1392-1395.

[BaGü01] Bauer, A.; Günzel, H. (Hrsg.): Data Warehouse Systeme. Architektur, Entwicklung, Anwendung. Heidelberg 2001.

[BDKK02] Becker, J.; Delfmann, P.; Knackstedt, R.; Kuropka, D.: Konfigurative Referenzmodellierung. In: Becker, J.; Knackstedt, R. (Hrsg.): Wissensmanagement mit Referenzmodellen. Konzepte für die Anwendungssystem- und Organisationsgestaltung. Heidelberg 2002, S. 25-144.

[BeHo98] Becker, J.; Holten, R.: Fachkonzeptionelle Spezifikation von Führungsinformationssystemen. Wirtschaftsinformatik, 40 (1998) 6, S. 483-492.

[BeKn02] Becker, J.; Knackstedt, R.: Prozess- und Informationsmanagement für das CRM. In: Ahlert, D.; Becker, J.; Knackstedt, R.; Wunderlich, M. (Hrsg.): Customer Relationship Management im Handel. Strategien, Konzepte, Erfahrungen. Berlin et al. 2002.

[BeKS02] Becker, J.; Knackstedt, R.; Serries, T.: Informationsportale für das Management: Integration von Data-Warehouse- und Content-Management-Systemen. In: Von Maur, E.; Winter, R. (Hrsg.): Vom Data Warehouse zum Corporate Knowledge Center. Proceedings der Data Warehousing 2002. Heidelberg 2002, S. 241-261.

[BeSc96] Becker, J.; Schütte, R.: Handelsinformationssysteme. Landsberg am Lech 1996.

[BHKU01] Böhnlein, M.; Holten, R.; Knackstedt, R.; Ulbrich-vom Ende, E.: Identifikation und Anwendung semantischer Modellbausteine für Managementsichten. In: Jablonski, S.; Kirn, S.; Plaha, M.; Sinz, E. J.; Ulbrich-vom Ende, A.; Weiß, G. (Hrsg.): Verteilte Informationssysteme auf der Grundlage von Objekten, Komponenten und Agenten. Proceedings der Verbundtagung VertIS 2001. Rundbrief der GI-Fachgruppe 5.10 MobIS, 8 (2001) 2, S. 75-96.

[Blas00] FIESTA: A Framework for Schema Evolution in Multidimensional Databases. Dissertation, Technische Universität München 2000.

[Böhn01] Böhnlein, M.: Konstruktion semantischer Data-Warehouse-Schemata. Wiesbaden 2001.

[Broc02] vom Brocke, J.: Referenzmodellierung. Gestaltung und Verteilung von Konstruktionsprozessen. Diss., Universität Münster 2002.

[Bulo96] Bulos, D.: A New Dimension. OLAP Database Design. Database Programming \& Design 9 (1996) 6, S. 33-37.

[CCG97] Centrale für Coorganisation GmbH (Hrsg.): Standard-Warenklassifikation für Verbrauchsgüter, Gebrauchsgüter, Investitionsgüter und Rohstoffe. 2. Aufl., Köln 1997.

[Espr89] ESPRIT Consortium AMICE: Open System Architecture for CIM. Berlin u. a. 1989. 
[FeLo02] Fettke, P.; Loos, P.: Der Referenzmodellkatalog als Instrument des Wissensmanagements: Methodik und Anwendung. In: Becker, J.; Knackstedt, R. (Hrsg.): Wissensmanagement mit Referenzmodellen. Konzepte für die Anwendungssystem- und Organisationsgestaltung. Heidelberg 2002, S. 1-24.

[FeYu00] Fernandez, E. B.; Yuan, X.: Semantic Analysis Patterns. In : A. H. F. Laender, S. W. Liddle, V. C. Storey (Hrsg.): Conceptual Modeling - ER 2000 - 19th International Conference on Conceptual Modeling, Salt Lake City, Utah, USA, October 9-12. Berlin et al. 2000, S. 183-195.

[Fran94] Frank, U.: Multiperspektivische Unternehmensmodellierung. Theoretischer Hintergrund und Entwurf einer objektorientierten Entwicklungsumgebung. München, Wien 1994.

[Fran99] Frank, U.: Eine Architektur zur Spezifikation von Sprachen und Werkzeugen für die Unternehmensmodellierung. In: E. Sinz (Hrsg.): Modellierung betrieblicher Informationssysteme. Proceedings der MobIS-Fachtagung 1999. Bamberg 1999, S. 154-169.

[GaG197] Gabriel, R.; Gluchowki, P.: Semantische Modellierungstechniken für multidimensionale Datenstrukturen. HMD, 34 (1997) 195, S. 18-37.

[Hahn02] Hahne, M.: Transformation mehrdimensionaler Datenmodelle. In: Von Maur, E.; Winter, R. (Hrsg.): Vom Data Warehouse zum Corporate Knowledge Center. Proceedings der Data Warehousing 2002. Heidelberg 2002, S. 399-420.

[Hars94] Hars, A.: Referenzdatenmodelle. Grundlagen effizienter Datenmodellierung. Wiesbaden 1994.

[HaTB02] Habermann, F.; Thomas, O.; Botta, C.: Organisational-Memory-System zur Unterstützung informationstechnisch basierter Verbesserungen von Geschäftsprozessen. In: Becker, J.; Knackstedt, R. (Hrsg.): Wissensmanagement mit Referenzmodellen. Konzepte für die Anwendungssystem- und Organisationsgestaltung. Heidelberg 2002, S. 291-322.

[HeHa99] Herden, O.; Harren, A.: MML und mUML - Sprache und Werkzeug zur Unterstützung des konzeptionellen Data Warehouse-Design. In: Proc. 2. GI-Workshop „Data Mining und Data Warehousing als Grundlage moderner entscheidungsunterstützender Systeme (DMDW'99). Magdeburg 1999.

[Herd01] Herden, O.: Eine Entwurfsmethodik für Data Warehouses. Diss., Universität Oldenburg 2001.

[HoDS02] Holten, R.; Dreiling, A.; Schmid, B.: Management Report Engineering - A Swiss Re Business Case. In: Von Maur, E.; Winter, R. (Hrsg.): Vom Data Warehouse zum Corporate Knowledge Center. Proceedings der Data Warehousing 2002. Heidelberg 2002, S. 421-437.

[HoKn99] Holten, R.; Knackstedt, R.: Fachkonzeptuelle Modellierung von Führungsinformationssystemen am Beispiel eines filialisierenden Einzelhandelsunternehmens. In: Sinz, E. J. (Hrsg.): Modellierung betrieblicher Informationssysteme. Proceedings der MobIS-Fachtagung 1999. Rundbrief der GI-Fachgruppe 5.10, 6 (1999) 1, S. 48-64.

[Holt99] Holten, R.: Entwicklung von Führungsinformationssystemen. Ein methodenorientierter Ansatz. Wiesbaden 1999. 
[KeNS92] Keller, G.; Nüttgens, M.; Scheer, A.-W.: Semantische Prozeßmodellierung auf der Grundlage „Ereignisgesteuerter Prozeßketten (EPK)“. Veröffentlichungen des Instituts für Wirtschaftsinformatik. Heft 89. Hrsg.: A.-W. Scheer. Saarbrücken 1992.

[Knac01] Knackstedt, R.: Konfigurative Referenzmodelle als Instrumente des Wissensmanagements bei der Data-Warehouse-Entwicklung. In: H.-P. Schnurr, S. Staab, R. Studer, G. Stumme, Y. Sure (Hrsg.): Professionelles Wissensmanagement. Erfahrungen und Visionen. Aachen 2001, S. 113-128.

[Kurz99] Kurz, A.: Data Warehousing. Enabling Technology. Bonn 1999.

[Lang97] Lang, K.: Gestaltung von Geschäftsprozessen mit Referenzprozessbausteinen. Wiesbaden 1997.

[Loud94] Louden, K. C.: Programmiersprachen: Grundlagen - Konzepte - Entwurf. Bonn et al. 1994.

[Maie96] Maier, R.: Qualität von Datenmodellen. Wiesbaden 1996.

[Matt96] Mattison, R.: Data Warehousing. Strategies, Technologies and Techniques. New York 1996.

[Meff91] Meffert, H.: Marketing. Grundlagen der Absatzpolitik. 7. Aufl., Nachdr., Wiesbaden 1991.

[MeLo00] Mertens, P.; Lohmann, M.: Branche oder Betriebstyp als Klassifikationskriterien für die Standardsoftware der Zukunft? Erste Überlegungen, wie künftig betriebswirtschaftliche Standardsoftware entstehen könnte. In: F. Bodendorf, M. Grauer (Hrsg.): Verbundtagung Wirtschaftsinformatik 2000. Aachen 2000, S. 110-135.

[MLEM99] Mertens, P.; Ludwig, P.; Engelhardt, A.; Möhle, S.; Kaufmann, T.; Ließmann, H.: Ausgewählte Experimente zu Mittelwegen zwischen Individual- und Standardsoftware. In: J. Becker, M. Rosemann, R. Schütte (Hrsg.): Referenzmodellierung. State-ofthe-Art und Entwicklungsperspektiven. Heidelberg 1999, S. 70-106.

[NSKL98] Nordstrom, G.; Sztipanovits, J.; Karsai, G.; Ledeezi, A.: Metamodeling - Rapid Design and Evolution of Domain-Specific Modeling Environments. Proc. IEEE, ECBS'98 Conf.

[Poe96] Poe, V.: Building a Data Warehouse for Decision Support. New Jersey 1996.

[Reic97] Reichmann, T.: Controlling mit Kennzahlen und Managementberichten. Grundlagen einer systemgestützten Controlling-Konzeption. 5. Aufl., München 1997.

[Remm97] Remme, M.: Konstruktion von Geschäftsprozessen. Ein modellgestützter Ansatz durch Montage generischer Prozesspartikel. Wiesbaden 1997.

[Risi00] Rising, L.: The Pattern Almanac 2000. Bosten et al. 2000.

[Roh195] Rohloff, M.: Produktionsmanagement in modularen Organisationsstrukturen. Reorganisation der Produktion und objektorientierte Informationssysteme für verteilte Planungssegmente. München, Wien 1995. 
[Rose98] Rosemann, M.: Grundsätze ordnungsmäßiger Modellierung. Intention, Entwicklung, Architektur und Multiperspektivität. In: Maicher, M.; Scheruhn, H.-J. (Hrsg.): Informationsmodellierung. Referenzmodelle und Werkzeuge. Wiesbaden 1998, S. 1-21.

[SBHD99] Sapia, C.; Blaschka, M.; Höfling, G.; Dinter, B.: Extending the E/R Model for the Multidimensional Paradigm. In: Advances in Database Technologies Lecture Notes in Computer Science, Vol. 1552. Berlin et. al. 1999, S. 105-116.

[Scha97] Scharl, A.: Referenzmodellierung kommerzieller Masseninformationssysteme. Idealtypische Gestaltung von Informationsangeboten im World Wide Web am Beispiel der Branche Informationstechnik. Frankfurt am Main 1997.

[Sche98] Scheer, A.-W.: ARIS - Vom Geschäftsprozeß zum Anwendungssystem. 3. Aufl., Berlin et al. 1998.

[Sche98] Schelp, J.: Konzeptionelle Modellierung mehrdimensionaler Datenstrukturen. In: P. Chamoni, P. Gluchowski (Hrsg.), Analytische Informationssysteme. Berlin, Heidelberg 1998, S. 263-276.

[Schü98] Schütte, R.: Grundsätze ordnungsmäßiger Referenzmodellierung. Konstruktion konfigurations- und anpassungsorientierter Modelle. Wiesbaden 1998.

[Schw99] Schwegmann, A.: Objektorientierte Referenzmodellierung. Theoretische Grundlagen und praktische Anwendung. Wiesbaden 1999.

[Simo98] Simoneit, M.: Informationsmanagement in Universitätsklinika. Konzeption und Implementierung eines objektorientierten Referenzmodells. Wiesbaden 1998.

[Spit97] Spitta, T.: Wiederverwendbare Attribute als Ausweg aus dem Datenchaos. HDM, 34 (1997) 195, S. 38-55.

[Stra96] Strahringer, S.: Metamodellierung als Instrument des Methodenvergleichs. Eine Evaluierung am Beispiel objektorientierter Analysemethoden. Aachen 1996.

[Stru97] Struckmeier, H.: Gestaltung von Führungsinformationssystemen. Betriebswirtschaftliche Konzeption und Softwareanforderungen. Wiesbaden 1997.

[StWi02] Strauch, B.; Winter, R.: Vorgehensmodell für die Informationsbedarfsanalyse im Data Warehousing. In: Von Maur, E.; Winter, R. (Hrsg.): Vom Data Warehouse zum Corporate Knowledge Center. Proceedings der Data Warehousing 2002. Heidelberg 2002, S. 359-378.

[Toto00] Totok, A: Multidimensionale Modellierung von OLAP- und Data-WarehouseSystemen. Wiesbaden 2000.

[Wolf01] Wolf, S.: Wissenschaftstheoretische und fachmethodische Grundlagen der Konstruktion von generischen Referenzmodellen betrieblicher Systeme. Aachen 2001.

[Woll86] Wollnik, M.: Implementierung computergestützter Informationssysteme. Berlin, New York 1986.

[Zach87] Zachman, J. A.: A Framework for Information Systems Architecture. IBM Systems Journal, 26 (1987) 3, S. 277-293. 